\title{
Gait trajectory generation for a five link bipedal robot based on a reduced dynamical model
}

\author{
${ }^{1}$ Yosra Arous \& ${ }^{2}$ Olfa Boubaker \\ ${ }^{1,2}$ National Institute of Applied sciences and Technology, \\ Tunis, Tunisia \\ 2olfa.boubaker@insat.rnu.tn
}

\begin{abstract}
In this paper, a simple trajectory generation method for biped walking is proposed. The dynamic model of the five link bipedal robot is first reduced using several biologically inspired assumptions. A sinusoidal curve is then imposed to the ankle of the swing leg's trajectory. The reduced model is finally obtained and solved: it is an homogeneous $2^{\text {nd }}$ order differential equations with constant coefficients. The algebraic solution obtained ensures a stable rhythmic gait for the bipedal robot. It's continuous in the defined time interval, easy to implement when the boundary conditions are well defined.
\end{abstract}

Index Terms - Trajectory Generation, Biped Locomotion, model reduction.

\section{INTRODUCTION}

Gait pattern generation [1] is one of key problems of research devoted to bipedal robots. Two kinds of works dedicated to the bipedal walking pattern generation can be distinguished: studies assimilating robots as elementary models and works considering all morphological data of the robot, see [2] and references therein. For the first approach, the linear inverted pendulum model concept is the mostly used concept in order to generate the gait trajectory [3, 4, 5]. For the second group of works attention is paid on the generation of a trajectory tracking control using objective function composed of one or more terms to minimize $[2,6,7]$.

In this paper, a simple trajectory generation method is proposed. The dynamic model of the five link bipedal robot is first reduced using several biologically inspired assumptions. A sinusoidal curve is then imposed to the ankle of the swing leg's trajectory. The reduced model is finally obtained and solved: it is an homogeneous $2^{\text {nd }}$ order differential equations with constant coefficients. The solution gives an algebraic solution for joint desired trajectories that ensures a stable rhythmic gait for the bipedal robot.

\section{THE FIVE LINK BIPEDAL ROBOT}

The planar bipedal robot prototype is composed of five links associated to five DOF. Fig.1 shows the involved rotations for each link. All physical parameters involved in the kinematic and dynamic models are given by Table 1 . They are inspired from [8]. The parameters $\mathrm{m}_{\mathrm{i}}, \mathrm{L}_{\mathrm{i}}, \mathrm{k}_{\mathrm{i}}$ and $\mathrm{I}_{\mathrm{i}},(i=1, . .5)$, design mass, length, position of center of mass and inertia about center of mass, respectively.

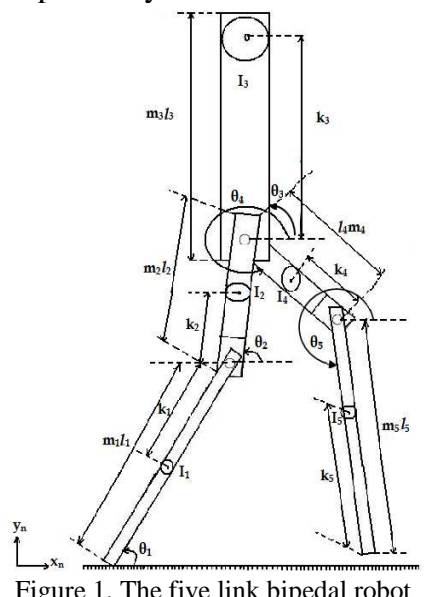

Table1. Physical parameters of the bipedal robot [8]

\begin{tabular}{|c|c|c|c|c|c|}
\hline link & Right leg & $\begin{array}{c}\text { Right } \\
\text { thigh }\end{array}$ & Pelvis & $\begin{array}{c}\text { Left } \\
\text { thigh }\end{array}$ & Left leg \\
\hline joint & $\begin{array}{c}\text { Right } \\
\text { ankle }\end{array}$ & $\begin{array}{c}\text { Right } \\
\text { knee }\end{array}$ & $\begin{array}{c}\text { Right } \\
\text { hip }\end{array}$ & $\begin{array}{c}\text { Left } \\
\text { hip }\end{array}$ & $\begin{array}{c}\text { Left } \\
\text { knee }\end{array}$ \\
\hline Link number & 1 & 2 & 3 & 4 & 5 \\
\hline Mass (kg) & 3.255 & 7.000 & 24.850 & 7.000 & 3.255 \\
\hline Lenght (m) & 0.426 & 0.424 & 0.299 & 0.424 & 0.426 \\
\hline $\begin{array}{l}\text { Center of } \\
\text { mass (kg.m) }\end{array}$ & 0.164 & 0.366 & 1.530 & 0.366 & 0.164 \\
\hline $\begin{array}{l}\text { Inertia about } \\
\text { centre of } \\
\text { mass (Kgm }\end{array}$ & 0.184 & 0.184 & 0.206 & 0.184 & 0.184 \\
\hline
\end{tabular}




\section{KINEMATIC AND DYNAMIC MODELING}

The five-link bipedal robotic system with five degrees of freedom can be described by the following direct kinematic model:

$X=h(\theta)$

where $\theta=\left[\begin{array}{lllll}\theta_{1} & \theta_{2} & \theta_{3} & \theta_{4} & \theta_{5}\end{array}\right]^{T} \in \mathfrak{R}^{5} \quad$ is the joint displacement vector, $X=\left[\begin{array}{ll}x & y\end{array}\right]^{T} \in \mathfrak{R}^{2}$ is the Cartesian position vector and $h(\theta) \in \mathfrak{R}^{2}$ is a nonlinear function described by:

$$
h(\theta)=\left[\begin{array}{c}
l_{1} \cos \theta_{1}+l_{2} \cos \theta_{2}-l_{4} \cos \theta_{4}-l_{5} \cos \theta_{5} \\
l_{1} \sin \theta_{1}+l_{2} \sin \theta_{2}+l_{4} \sin \theta_{4}+l_{5} \sin \theta_{5}
\end{array}\right]
$$

The time derivative of the direct kinematic model (1) yields the following differential kinematic model:

$$
\dot{X}=J(\theta) \dot{\theta}
$$

where $\dot{X}=\left[\begin{array}{ll}\dot{x} & \dot{y}\end{array}\right]^{T}$ is the Cartesian velocity vector, $\dot{\theta}=\left[\begin{array}{lllll}\dot{\theta}_{1} & \dot{\theta}_{2} & \dot{\theta}_{3} & \dot{\theta}_{4} & \dot{\theta}_{5}\end{array}\right]^{T}$ is the vector of joint velocities and $J(\theta)$ is the so-called analytical Jacobian matrix given by:

$J(\theta)=\left(\begin{array}{ccccc}-l_{1} \sin \theta_{1} & -l_{2} \sin \theta_{2} & 0 & l_{4} \sin \theta_{4} & l_{5} \sin \theta_{5} \\ l_{1} \cos \theta_{1} & l_{2} \cos \theta_{2} & 0 & l_{4} \cos \theta_{4} & l_{5} \cos \theta_{5}\end{array}\right)$

A typical walking cycle may include three phases [1]: the single support phase (SSP), the impact phase (IP) and the double support phase (DSP), (see Fig.2). In this paper we focus only on SSP.

Using Lagrange approach [9], the dynamical model of the bipedal robot in SSP phase is described by:

$$
M(\theta) \ddot{\theta}+H(\theta, \dot{\theta})+G(\theta)=D U
$$

where $\ddot{\theta}=\left[\begin{array}{lllll}\ddot{\theta}_{1} & \ddot{\theta}_{2} & \ddot{\theta}_{3} & \ddot{\theta}_{4} & \ddot{\theta}_{5}\end{array}\right]^{T} \quad$ is the vector of joint accelerations, $U=\left[\begin{array}{lllll}U_{1} & U_{2} & U_{3} & U_{4} & U_{5}\end{array}\right]^{T}$ is the vector of torque inputs, $M(\theta)$ is the symmetric positive definite inertia matrix, $H(\theta, \dot{\theta})$ is the vector of centripetal and Coriolis torques and $G(\theta)$ is the vector of gravitational torques given by $M(\theta)=\left[\left.M_{i j}\right|_{1 \leq i \leq 5 \leq 5}, \quad H(\theta, \dot{\theta})=\left[h_{i j}\right]_{1 \leq i \leq 5}, \quad G(\theta)=\left[G_{i}\right]_{1 \leq i \leq 5}\right.$ where:

$$
\begin{aligned}
& M_{11}=m_{1} k_{1}^{2}+I_{1}+\left(m_{2}+m_{3}+m_{4}+m_{5}\right) l_{1}^{2} \\
& M_{12}=\left(m_{2} l_{1} k_{2}+m_{3} l_{1} l_{2}+m_{4} l_{1} l_{2}+m_{5} l_{1} l_{2}\right) \cos \left(\theta_{1}-\theta_{2}\right) \\
& M_{13}=m_{3} l_{1} k_{3} \cos \left(\theta_{1}-\theta_{3}\right) \\
& M_{14}=\left(m_{4} l_{1}\left(l_{4}-k_{4}\right)\right)+m_{5} l_{1} l_{5} \cos \left(\theta_{1}+\theta_{5}\right) \\
& M_{15}=m_{5} l_{1}\left(l_{5}-k_{5}\right) \cos \left(\theta_{1}+\theta_{5}\right) \\
& M_{21}=\left(m_{2} l_{1} k_{2}+\left(m_{3}+m_{4}+m_{5}\right) l_{1} l_{2}\right) \cos \left(\theta_{1}-\theta_{2}\right) \\
& M_{22}=m_{2} k_{2}^{2}+I_{2}+\left(m_{3}+m_{4}+m_{5}\right) l_{2}^{2} \\
& M_{23}=m_{3} l_{2} k_{3} \cos \left(\theta_{2}-\theta_{3}\right) \\
& M_{24}=\left(m_{4} l_{2}\left(l_{4}-k_{4}\right)+m_{5} l_{2} l_{4} \cos \left(\theta_{2}+\theta_{4}\right)\right. \\
& M_{25}=m_{5} l_{2}\left(l_{5}-k_{5}\right) \cos \left(\theta_{2}+\theta_{5}\right)
\end{aligned}
$$

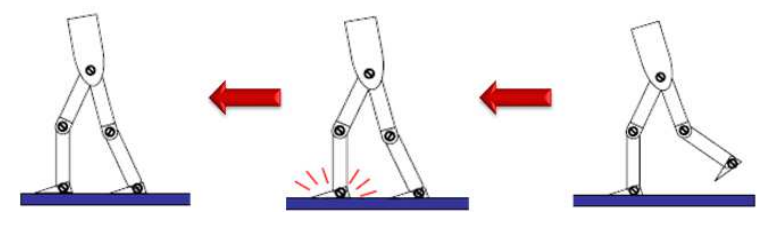

Figure 2.The three phases of a typical walking cycle

$$
\begin{aligned}
& M_{31}=m_{3} l_{1} k_{3} \cos \left(\theta_{1}-\theta_{3}\right) \\
& M_{32}=m_{3} l_{2} k_{3} \cos \left(\theta_{2}-\theta_{3}\right) \\
& M_{33}=m_{3} k_{3}^{2}+I_{3} \\
& M_{34}=J_{35}=0 \\
& M_{41}=\left(m_{4} l_{1}\left(l_{4}-k_{4}\right)+m_{5} l_{1} l_{4}\right) \cos \left(\theta_{1}+\theta_{4}\right) \\
& M_{42}=\left(m_{4} l_{2}\left(l_{4}-k_{4}\right)+m_{5} l_{2} l_{4}\right) \cos \left(\theta_{2}+\theta_{4}\right) \\
& M_{43}=0 \\
& M_{44}=m_{4}\left(l_{4}-k_{4}\right)^{2}+m_{5} l_{4}^{2}+I_{4} \\
& M_{45}=\left(m_{5} l_{4}\left(l_{5}-k_{5}\right)\right) \cos \left(\theta_{4}-\theta_{5}\right) \\
& M_{51}=m_{5} l_{1}\left(l_{5}-k_{5}\right) \cos \left(\theta_{1}+\theta_{5}\right) \\
& M_{52}=m_{5} l_{2}\left(l_{5}-k_{5}\right) \cos \left(\theta_{2}+\theta_{5}\right) \\
& M_{53}=0 \\
& M_{54}=m_{5} l_{4}\left(l_{5}-k_{5}\right) \cos \left(\theta_{4}-\theta_{5}\right) \\
& M_{55}=m_{5}\left(l_{5}-k_{5}\right)^{2}+I_{5} \\
& h_{11}=0 \\
& h_{12}=\left(m_{2} l_{1} k_{2}+m_{3} l_{1} l_{2}+m_{4} l_{1} l_{2}+m_{5} l_{1} l_{2}\right) \sin \left(\theta_{1}-\theta_{2}\right) \\
& h_{13}=\left(m_{5} l_{1} k_{3}\right) \sin \left(\theta_{1}-\theta_{3}\right) \\
& h_{14}=-\left(m_{4} l_{1}\left(l_{4}-k_{4}\right)+m_{5} l_{1} l_{4}\right) \sin \left(\theta_{1}+\theta_{4}\right) \\
& h_{15}=-\left(m_{5} l_{1}\left(l_{5}-k_{5}\right)\right) \sin \left(\theta_{1}+\theta_{5}\right) \\
& h_{21}=-\left(m_{2} l_{1} k_{2}+\left(m_{3}+m_{4}+m_{5}\right) l_{1} l_{2}\right) \sin \left(\theta_{1}-\theta_{2}\right) \\
& h_{22}=0 \\
& h_{23}=m_{3} l_{2} k_{3} \sin \left(\theta_{2}-\theta_{3}\right) \\
& h_{24}=-\left(m_{4} l_{2}\left(l_{4}-k_{4}\right)+m_{5} l_{2} l_{4}\right) \sin \left(\theta_{2}+\theta_{4}\right) \\
& h_{25}=-m_{5} l_{2}\left(l_{5}-k_{5}\right) \sin \left(\theta_{2}+\theta_{5}\right) \\
& h_{31}=-m_{3} l_{1} k_{3} \sin \left(\theta_{1}-\theta_{3}\right) \\
& h_{32}=-m_{3} l_{2} k_{2} \sin \left(\theta_{2}-\theta_{3}\right) \\
& h_{33}=0 \\
& h_{34}=h_{35}=0 \\
& h_{41}=-\left(m_{4} l_{1}\left(l_{4}-k_{4}\right)+m_{5} l_{1} l_{4}\right) \sin \left(\theta_{1}+\theta_{4}\right) \\
& h_{42}=-\left(m_{4} l_{2}\left(l_{4}-k_{4}\right)+m_{5} l_{2} l_{4}\right) \sin \left(\theta_{2}+\theta_{4}\right) \\
& h_{43}=0 \\
& h_{44}=0 \\
& h_{45}=\left(m_{5} l_{4}\left(l_{5}-k_{5}\right)\right) \sin \left(\theta_{4}-\theta_{5}\right) \\
& h_{51}=-\left(m_{5} l_{1}\left(l_{5}-k_{5}\right)\right) \sin \left(\theta_{1}+\theta_{5}\right) \\
& h_{52}=-\left(m_{5} l_{2}\left(l_{5}-k_{5}\right)\right) \sin \left(\theta_{2}+\theta_{5}\right) \\
& h_{53}=h_{55}=0 \\
& h_{54}=-\left(m_{5} l_{4}\left(l_{5}-k_{5}\right)\right) \sin \left(\theta_{4}-\theta_{5}\right)
\end{aligned}
$$


$G_{1}=g\left(m_{1} k_{1}+\left(m_{2}+m_{3}+m_{4}+m_{5}\right) l_{1}\right) \cos \theta_{1}$

$G_{2}=g\left(m_{2} k_{2}+\left(m_{3}+m_{4}+m_{5}\right) l_{2}\right) \cos \theta_{2}$

$G_{3}=m_{3} g k_{3} \cos \theta_{3}$

$G_{4}=g\left(m_{4}\left(l_{4}-k_{4}\right)+m_{5} l_{4}\right) \cos \theta_{4}$

$G_{5}=g\left(m_{5}\left(l_{5}-k_{5}\right)\right) \cos \theta_{5}$

$D=\left(\begin{array}{ccccc}1 & -1 & 0 & 0 & 0 \\ 0 & 1 & -1 & 0 & 0 \\ 0 & 0 & 1 & -1 & 0 \\ 0 & 0 & 0 & 1 & -1 \\ 0 & 0 & 0 & 0 & 1\end{array}\right)$

\section{Model Reduction AND GaIt TRAJECTORy GENERATION}

The purpose of this section is to reduce the nonlinear dynamic model (3) into a solvable differential system in order to give algebraic solutions of gait trajectories. The reduced model will be obtained as follows: the robotic system (3) is first written around an equilibrium point $\theta_{e q}$ as [9]:

$\dot{x}=A x+B v$

$$
\begin{aligned}
& A=\left[\begin{array}{cc}
0_{5 \times 5} & I_{5 \times 5} \\
-\left.J^{-1} \frac{\partial G}{\partial \theta}\right|_{e q} & 0_{5 \times 5}
\end{array}\right] \quad B=\left[\begin{array}{c}
0_{5 \times 5} \\
J^{-1} D
\end{array}\right] \\
& v=\left[\begin{array}{ll}
0_{1 \times 5} & U-U_{e q}
\end{array}\right]^{T} \\
& x=\left[\begin{array}{ll}
\theta-\theta_{e q} & \dot{\theta}-\dot{\theta}_{e q}
\end{array}\right]^{T}
\end{aligned}
$$

To generate joint desired joint trajectory vector:

$$
\theta_{d}(t)=\left[\begin{array}{lllll}
\theta_{1, d}(t) & \theta_{2, d}(t) & \theta_{3, d}(t) & \theta_{4, d}(t) & \theta_{5, d}(t)
\end{array}\right]^{T}
$$

we assume, for the bipedal robot, the following biologically inspired assumptions (see Fig.3):

Assumption 1: ZMP stability [10] is imposed. This is can be ensured by assuming that:

$U_{1}=0$

Assumption 2: the supporting leg is kept straight as:

$$
\theta_{1, d}(t)=\theta_{2, d}(t)
$$

Assumption 3: The bipedal robot must have an upright posture that is to say, it keeps its back straight such that:

$$
\theta_{3, d}(t)=\frac{\pi}{2}
$$

Assumption 4: the relationship between the right ankle joint and the left hip joint is imposed such that:

$\theta_{4, d}(t)=\theta_{1, d}(t)+\alpha$

where $\alpha$ is a given constant angle to be chosen.

Assumption 5: To generate a rhythmic stable movement, the relationship between the right ankle joint and the left knee joint is given by [11]:

$$
\theta_{5, d}(t)=\theta_{1, d}(t)-\sin ^{2}\left(\frac{\pi}{T} t\right)
$$

From the assumptions (6)-(9), we can then easily deduce the desired velocity vector and the desired acceleration vector defined, respectively, by:

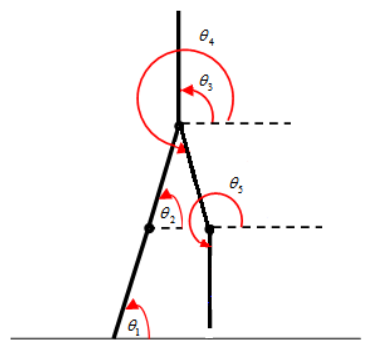

Figure 3. Biologically inspired assumptions

$$
\begin{aligned}
& \dot{\theta}_{d}(t)=\left[\begin{array}{lllll}
\dot{\theta}_{1, d}(t) & \dot{\theta}_{2, d}(t) & \dot{\theta}_{3, d}(t) & \dot{\theta}_{4, d}(t) & \dot{\theta}_{5, d}(t)
\end{array}\right]^{T} \\
& \ddot{\theta}_{d}(t)=\left[\begin{array}{lllll}
\ddot{\theta}_{1, d}(t) & \ddot{\theta}_{2, d}(t) & \ddot{\theta}_{3, d}(t) & \ddot{\theta}_{4, d}(t) & \ddot{\theta}_{5, d}(t)
\end{array}\right]^{T}
\end{aligned}
$$

Particularly the following relations are deduced:

$\ddot{\theta}_{1, d}(t)=\ddot{\theta}_{2, d}(t)$

$\ddot{\theta}_{3, d}(t)=0$

$\ddot{\theta}_{4, d}(t)=\ddot{\theta}_{1, d}(t)$

$\ddot{\theta}_{5, d}=\ddot{\theta}_{1, d}+2\left(\frac{\pi}{T}\right)^{2} \cos \left(2 \frac{\pi}{T} t\right)$

By summing all the lines of the system (4) and using the relation (5), (10)-(13), the following reduced homogeneous $2^{\text {nd }}$ order differential equations with constant coefficients is obtained as:

$$
M_{1} \ddot{\theta}_{1, d}(t)+H_{1} \theta_{1, d}(t)=-K-h(t)-s(t)
$$

where:

$$
\begin{aligned}
& M_{1}=\left.\left(M_{11}+M_{22}+M_{44}+M_{55}+M_{23}\right)\right|_{\theta_{e q}}+\left.2\left(M_{12}+M_{14}+M_{15}+M_{25}+M_{45}\right)\right|_{\theta_{e q}} \\
& H_{1}=\left.\left(\frac{\partial G_{1}}{\partial \theta_{1}}+\frac{\partial G_{2}}{\partial \theta_{2}}+\frac{\partial G_{4}}{\partial \theta_{4}}+\frac{\partial G_{5}}{\partial \theta_{5}}\right)\right|_{\theta_{e q}} \\
& K=-\left.\left(\frac{\partial G_{1}}{\partial \theta_{1}} \theta_{1}+\frac{\partial G_{2}}{\partial \theta_{2}} \theta_{2}+\frac{\partial G_{3}}{\partial \theta_{3}} \theta_{3}+\frac{\partial G_{4}}{\partial \theta_{4}} \theta_{4}+\frac{\partial G_{5}}{\partial \theta_{5}} \theta_{5}\right)\right|_{\theta_{e q}} \\
& \quad+\left.\left(\alpha \frac{\partial G_{4}}{\partial \theta_{4}}+\frac{\pi}{2} \frac{\partial G_{3}}{\partial \theta_{3}}+\frac{1}{2} \frac{\partial G_{5}}{\partial \theta_{5}}\right)\right|_{\theta_{e q}}+U_{1 e q} \\
& h(t)=-\frac{\partial G_{5}}{2 \partial \theta_{5}}\left(\cos 2 \frac{\pi}{T} t\right) \\
& s(t)=2\left(\frac{\pi}{T}\right)^{2} M_{2} \cos \left(2 \frac{\pi}{T} t\right) \\
& M_{2}=M_{15}+M_{25}+M_{45}+M_{55}
\end{aligned}
$$

Solving (14) for the boundary conditions:

$$
\begin{gathered}
\theta_{1, d}\left(t_{0}\right)=\theta_{10} \\
\theta_{1, d}\left(t_{f}\right)=\theta_{1 f}
\end{gathered}
$$


and for the equilibrium point :

$\theta_{e q}=\left[\begin{array}{lllll}\frac{\pi}{2} & \frac{\pi}{2} & \frac{\pi}{2} & \frac{\pi}{2} & \frac{\pi}{2}\end{array}\right]^{T}$

the analytical solution of the differential equation (14) is given by [12]:

$\theta_{1, d}(t)=C_{1} e^{r_{1} t}+C_{2} e^{r_{2} t}+C_{3} \cos \left(2 \frac{\pi}{T} t\right)-\frac{K}{H_{1}}$

where :

$C_{1}=C_{2}-\frac{K}{H_{1}}+C_{3}-\theta_{10}$

$C_{2}=\frac{\left(C_{3}+\theta_{1 f}-\theta_{10}-\frac{K}{H_{1}}\right) e^{r_{1} t_{f}}-C_{3} \cos \left(2 \frac{\pi}{T} t_{f}\right)+K / H_{1}}{e^{r_{1} t_{f}}+e^{r_{2} t_{f}}}$

$C_{3}=\frac{\frac{\partial G_{5}}{2 \partial \theta_{5}}-M_{2}\left(\frac{\pi}{T}\right)^{2}}{\left(4\left(\frac{\pi}{T}\right)^{2} M_{1}-H_{1}\right)}$

$r_{1,2}= \pm \frac{\sqrt{-M_{1} \cdot H_{1}}}{M_{1}}, M_{1} H_{1}<0$

To verify the algebraic solution (15) that asked a rather tedious calculation two approaches are used: a symbolic computation of the solution (15) using the symbolic toolbox of Matlab software and a numerical integration of the differential equation (14) using the ode45 function of the same software. The three solutions were found superimposed for the physical parameters given by Table 1 .

Using the relations (15) and (6)-(9), the analytical expressions of the joint trajectories are then deduced. We impose then to the robotic model (3) the following second order linear inputoutput behavior [8]:

$$
\left(\ddot{\theta}(t)-\ddot{\theta}_{d}(t)\right)+K_{v}\left(\dot{\theta}(t)-\dot{\theta}_{d}(t)\right)+K_{p}\left(\theta(t)-\theta_{d}(t)\right)=0
$$

where $K_{v} \in \mathfrak{R}^{5 \times 5}$ and $K_{p} \in \mathfrak{R}^{5 \times 5}$ are two positive definite diagonal matrices chosen to guarantee global stability, desired performances and decoupling proprieties for the controlled system and such that the desired trajectories $\theta_{d}, \dot{\theta}_{d}$ and are derived from the solution (15) and the relations (6)-(9). The control law deduced from (3) and (16) is then given by:

$$
\begin{array}{r}
U=D^{-1}\left[M(\theta)\left(\ddot{\theta}_{d}(t)-K_{v}\left(\dot{\theta}(t)-\dot{\theta}_{d}(t)\right)-K_{p}\left(\theta(t)-\theta_{d}(t)\right)\right)\right. \\
+H(\theta, \dot{\theta})+G(\theta)]
\end{array}
$$

Using the physical parameters given in table1, the joint trajectories of the controlled biped robot are generated as see by Fig. 4 where the desired joint position is designed by

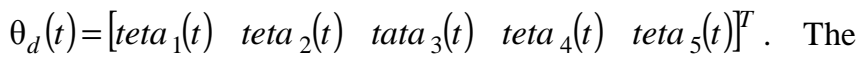
walking cycle is shown by Fig.5.

Compared to previous works [8], this paper gives an algebraic solution for desired joint trajectories that must be followed by the robotic system. The result is a stable rhythmic movement for the planar bipedal system.

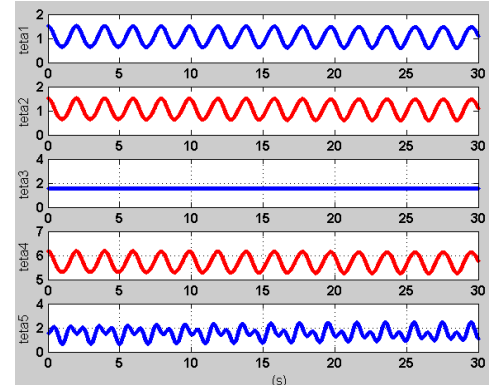

Figure 4. Joint trajectories of the bipedal robot in the swing phase

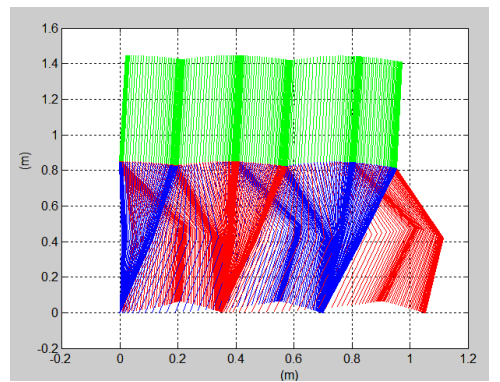

Figure 5. The resulting walking cycle

\section{CONCLUSION}

A simple trajectory generation method is proposed for biped al gait by imposing a sinusoidal curve to the ankle of the swing leg's trajectory.

\section{REFERENCES}

[1] M. Vukobratovic, B. Borovac, D. Surla, D. Stokic "Biped locomotion: dynamics, stability, control and application," Springer-Verlag, 1989.

[2] A. Aloulou, O. Boubaker, "Minimum jerk-based control for a three dimensional bipedal robot," Lecture notes in Computer Science, Vol. 7102, 2011, pp. 251-262.

[3] K. Erbatur, U. Seven, "An inverted pendulum based approach to biped trajectory generation with swing leg dynamics," Proc. IEEE International Conference on Humanoid Robots, November 2007, pp. 216-221.

[4] N. Motoi, T. Suzuki, K. Ohnishi, “A bipedal locomotion planning based on virtual linear inverted pendulum model," IEEE Transactions on Industrial Electronics, vol. 56, January 2009, pp. $54-61$.

[5] S. Kajita, F. Kanehiro, K. Kaneko, K. Yokoi, H. Hirukawa, "The 3D linear inverted pendulum mode: A Simple Modeling for a Biped Walking Pattern Generation," Proc. IEEE International Conference on Intelligent Robots and Systems, vol. 1, 2001, pp. 239-246.

[6] Y. Hurmuzlu, "Dynamics of bipedal gait: Part I-objective functions and the contact event of a planar five-link biped," Journal of Applied Mechanics, vol. 60, June 1993, 331-336.

[7] C. Chevallereau, Y. Aoustin, "Optimal reference trajectories for walking and running of a biped robot," Robotica, vol. 19, August 2001, 557569.

[8] A. Aloulou, O. Boubaker, "Control of a step walking combined to arms swinging for a three dimensional humanoid prototype," Journal of Computer Science, vol. 6, August 2010, 886-895.

[9] M. Spong and M. Vidyasagar, "Robot dynamics and control," John Wiley \& Sons, New York, 1989.

[10] M. Vukobratovic, AA. Frank, D. Juricic, 1970, "On the stability of biped locomotion," IEEE Transactions on Biomedical Engineering, vol. BME17, 1970, pp. 25-36.

[11]T. Furuta, T. Tawara, Y. Okumura \& M. Shimizu. "Design and construction of series of compact humanoid robots and development of biped walk control strategies," Robotics and autonomous Systems, vol. 34, November 2001, pp. 81-100.

[12] E. Hairer, G. Wanner, "Solving ordinary differential equations II: Stiff and differential-algebraic problems," Springer-Verlag, 2010. 